\title{
Qualidade de vida em pacientes com Dispositivos de Assistência Ventricular de longa
}

\section{permanência}

\author{
Quality of life in patients with long-term Ventricular Assist Devices \\ Calidad de vida em pacientes con Dispositivos de Asistencia Ventricular de largo plazo
}

Adryel Vieira Caetano da Silva

ORCID: https://orcid.org/0000-0002-3472-8505 Hospital Sírio-Libanês, Brasil

E-mail: adryelvieira@hotmail.com

Eliane Laranjeira Saraiva

ORCID: https://orcid.org/0000-0002-1775-6758 Hospital Sírio-Libanês, Brasil

E-mail: elsaraiva89@gmail.com

Natalia Balestra

ORCID: https://orcid.org/0000-0002-2618-0113 Hospital Sírio-Libanês, Brasil

E-mail: natalia.balestra@hsl.org.br

\begin{abstract}
Resumo
Para a Insuficiência Cardíaca avançada e refratária à terapêutica clínica, o transplante cardíaco é o padrão-ouro do tratamento. Contudo, devido à dificuldade de doadores disponíveis e captação de órgãos viáveis, emergiram os Dispositivos de Assistência Ventricular (DAV), que oferecem um suporte circulatório mecânico como método alternativo para esses pacientes visando melhoria na qualidade de vida e taxa de sobrevivência. O estudo objetivou identificar, na literatura científica, a perspectiva sobre a qualidade de vida de pacientes que utilizam DAV e seus impactos, em estudos publicados no âmbito nacional e internacional. Trata-se de revisão integrativa de literatura de análise narrativa, realizada em bases de dados nacionais e internacionais utilizando a estratégia PICO. A busca foi feita com auxílio de uma profissional bibliotecária e a seleção foi feita em pares por dois pesquisadores obtendo uma amostra final de 21 estudos. Existem fatores positivos e negativos que devem ser considerados com o histórico, condição clínica, terapêutica, motivação, medo, enfrentamento, suporte emocional e social, dentre outros. Pacientes em uso de DAV apresentam melhora na sintomatologia, diminuem a descompensação clínica decorrente da insuficiência cardíaca e, consequentemente, melhoram a qualidade de vida.
\end{abstract}

Palavras-chave: Dispositivo de assistência ventricular; Qualidade de vida; Cardiologia.

\begin{abstract}
For advanced heart failure refractory to clinical therapy, heart transplantation is the gold standard of treatment. However, due to the difficulty of available donors and the capture of viable organs, Ventricular Assistance Devices (VAD) emerged, offering mechanical circulatory support as an alternative method for these patients, aiming to improve quality of life and survival rate. The study aimed to identify, in the scientific literature, the perspective on the quality of life of patients who use VAD and its impacts, in studies published nationally and internationally. This is an integrative literature review of narrative analysis, carried out in national and international databases using the PICO strategy. The search was carried out with the help of a professional librarian and the selection was made in pairs by two researchers, obtaining a final sample of 21 studies. There are positive and negative factors that must be considered with the history, clinical condition, therapy, motivation, fear, coping, emotional and social support, among others. Patients using VAD show improvement in symptoms, reduce clinical decompensation resulting from heart failure and, consequently, improve quality of life.
\end{abstract}

Keywords: Ventricular assist device; Quality of life; Cardiology.

\section{Resumen}

Para la insuficiencia cardíaca avanzada refractaria a la terapia clínica, el trasplante de corazón es el tratamiento de referencia. Sin embargo, debido a la dificultad de los donantes disponibles y la captura de órganos viables, surgieron los Dispositivos de Asistencia Ventricular (DAV), que ofrecen soporte circulatorio mecánico como un método alternativo para estos pacientes, con el objetivo de mejorar la calidad de vida y la tasa de supervivencia. El estudio tuvo como objetivo identificar, en la literatura científica, la perspectiva sobre la calidad de vida de los pacientes que usan DAV y sus impactos, en estudios publicados a nivel nacional e internacional. Se trata de una revisión bibliográfica integradora de análisis narrativo, realizada en bases de datos nacionales e internacionales utilizando la 
estrategia PICO. La búsqueda se realizó con la ayuda de un bibliotecario profesional y la selección fue realizada por parejas por dos investigadores, obteniendo una muestra final de 21 estudios. Existen factores positivos y negativos que deben ser considerados con la historia, condición clínica, terapia, motivación, miedo, afrontamiento, apoyo emocional y social, entre otros. Los pacientes que utilizan DAV muestran una mejoría de los síntomas, reducen la descompensación clínica derivada de la insuficiencia cardíaca y, en consecuencia, mejoran la calidad de vida.

Palabras clave: Dispositivo de asistencia ventricular; Calidad de vida; Cardiología.

\section{Introdução}

As doenças de etiologia cardiovascular configuram uma das principais problemáticas de saúde no cenário mundial e atualmente são consideradas uma das maiores causas de mortalidade dentro do sistema de saúde brasileiro (Ayub-Ferreira et al., 2016). Dentre as doenças cardiovasculares mais prevalentes, destaca-se a Insuficiência Cardíaca (IC) como uma complexa síndrome multifatorial resultante da incapacidade estrutural muscular contrátil das câmaras ventriculares em realizar a ejeção sanguínea necessária para atender às demandas fisiometabólicas corporais (Sousa et al., 2017).

As pessoas com IC possuem alterações no seu cotidiano e nas suas atividades diárias devido às limitações causadas pela condição. A New York Heart Association (NYHA) possui uma classificação funcional que auxilia na categorização dos pacientes com IC avaliando o grau crescente de limitações. São quatro classes funcionais (CF): I - assintomáticos e sem limitações nas atividades; II - sintomas leves e presença de dispneia em atividades cotidianas; III - sintomas moderados e presença de dispneia em atividades menos intensas que as cotidianas ou aos mínimos esforços e IV - dispneia em repouso (Rohde et al., 2018).

A American College of Cardiology (ACC)e a American Heart Association (AHA) classificam a IC em estágios correspondentes ao caráter progressivo da condição, sendo eles: A - Risco para desenvolver a IC, sem doenças estruturais ou sintomas da condição; B - Doença estrutural cardíaca presente, porém assintomático para IC; C - Presença de doença cardíaca estrutural, com sintomas prévios e/ou atuais da IC; D - IC refratária ao tratamento clínico com necessidade de intervenção especializada (Garlet et al., 2017).

Com isso, portadores de insuficiência cardíaca avançada refratários à otimização terapêutica padrão, caracterizado pela persistência da sintomatologia clínica (ou seja, estágio D), possuem o transplante cardíaco como padrão-ouro de alternativa terapêutica. Contudo, devido à dificuldade quanto a oferta de doadores disponíveis e captação de órgãos viáveis, o cenário é marcado por longas filas de transplante. A despeito desse cenário, emergiram os Dispositivos de Assistência Circulatória Mecânica (DACM), que oferecem um suporte circulatório mecânico como método alternativo para esses pacientes visando melhoria na qualidade de vida, taxa de sobrevivência e capacidade funcional (Jakovlievic et al., 2017; Slaughter \& Singh, 2012).

Uma das opções de DACM são os Dispositivos de Assistência Ventricular (DAV) de longa permanência. Esses dispositivos são caracterizados como bombas de fluxo contínuo com o objetivo de realizar suporte hemodinâmico para pacientes com IC, sendo alimentados por uma linha de transmissão (driveline) acoplada a uma bateria/fonte de energia externa e pode ser usado como ponte para transplante, recuperação miocárdica ou terapia de destino (Abshire et al., 2016). Os DAV visam a melhoria do funcionamento ventricular com o aumento do débito cardíaco, gerando repercussões hemodinâmicas favoráveis à funcionalidade cardíaca, diminuindo a sintomatologia da IC e melhorando a Qualidade de Vida (QV) dos pacientes (Shah et al., 2018).

A QV tem um conceito subjetivo que envolve entender como a pessoa reflete o seu estado de satisfação com a vida e pode ser caracterizada em como a mesma percebe o impacto de um tratamento e/ou uma condição clínica no seu dia-a-dia (MacIver \& Ross, 2012). Com isso, compreender as perspectivas de como os pacientes em uso de DAV percebem sua QV, tanto de forma positiva quanto negativa, impacta diretamente no cuidado prestado. 
Frente a isso, sabe-se que a melhoria da qualidade de vida de pacientes com IC avançada é pauta constante de estudos e o uso de DAV pode ser preponderante nesse aspecto. A QV depende de inúmeros fatores biopsicossociais que exercem influência na análise, mas que auxiliam na construção de um olhar assistencial focado nas necessidades dessa população (Barbosa et al., 2014).

Visando o conhecimento que pode ser agregado ao desenvolvimento deste estudo, fica implícita a relevância como forma de contribuir para a propagação de trabalhos acerca qualidade de vida de pacientes com IC avançada em uso de DAV.

Nesse sentido, questiona-se, "Qual a literatura existente sobre a qualidade de vida de pacientes que utilizam os Dispositivos de Assistência Ventricular de longa permanência?”. O estudo objetivou identificar, na literatura científica nacional e internacional, a perspectiva sobre a qualidade de vida de pacientes que utilizam Dispositivos de Assistência Ventricular (DAV) e seus impactos, em estudos publicados no âmbito nacional e internacional.

\section{Metodologia}

Trata-se de uma revisão integrativa de literatura de análise narrativa realizada em bases de dados nacionais e internacionais, pois a mesma traz contribuições para um maior aprofundamento de conhecimento sobre o tema devido à realização de levantamento de dados em estudos já existentes (Souza, Silva \& Carvalho, 2010).

Aplicou-se os seis passos propostos para a revisão: a identificação do tema e elaboração da questão de pesquisa, estabelecimento de critérios de inclusão e exclusão, identificação dos estudos nas bases de dados, categorização da amostra selecionada, análise e discussão dos resultados e apresentação da revisão (Cerqueira et al., 2018).

A metodologia de busca adotada foi a conhecida pelo acrômio PICO, em que P (patient) significa paciente; I (intervention) - intervenção; C (comparison group) - grupo de comparação e O (outcome) - resultado (Santos, Pimenta \& Nobre, 2007). A estratégia utilizada definiu como P - Pacientes com IC em estágio D, I - Uso de dispositivos de assistência ventricular, $\mathrm{C}$ - não se aplica e $\mathrm{O}$ - Perspectivas sobre a qualidade de vida.

O levantamento ocorreu no período compreendido entre os meses de julho a outubro de 2021 através das bases de dados Biblioteca Virtual em Saúde (BVS), Cumulative Index to Nursing and Allied Health Literature (CINAHL), Medical Literature Analysis and Retrieval System Online (MEDLINE) através da National Library of Medicine and National Institutes of Health (PubMed), Literatura Latino-Americana e do Caribe em Ciências da Saúde (LILACS) e SciVerse Scopus (Elsevier).

Os descritores inseridos na seleção da amostra foram elencados conforme os Descritores do Medical Subject Headings Section (MeSH): Quality of Life, Heart Artificial e/ou Heart-assist devices, tendo sido combinados através da utilização dos operadores booleanos "and" e "or".

Como critérios de inclusão para o estudo considerou-se: artigos disponíveis na íntegra, publicados eletronicamente, sem período temporal definido, redigidos nas línguas português, inglês e espanhol e que atendam a pergunta norteadora do estudo. Foram excluídos estudos duplicados, revisões, teses, dissertações e/ou relatos de experiências. A busca foi realizada com o auxílio de uma profissional bibliotecária para o cruzamento dos dados e acesso as bases de dados definidas.

A estratégia para o levantamento foi adaptada conforme a especificidade de cada base de dados envolvendo a utilização dos descritores e uso de caracteres especiais como aspas e/ou parêntesis. Para as bases de dados MEDLINE, CINAHL e LILACS, a busca foi realizada através da seguinte estratégia: (Heart-Assist Devices[MeSH Terms]) AND ("quality of life"[MeSH Terms]), tendo sido necessário o recorte temporal, na base de dados MEDLINE, dos últimos sete anos (20152021) para a obtenção de amostra para análise visto que o período anterior a esse não apresentou estudos que atendesse aos critérios.

A estratégia adotada pela base de dados SCOPUS foi: (KEY(heart-assist AND devices) AND KEY (quality AND of AND life)) AND ( LIMIT-TO (OA,"all")) AND (LIMIT-TO (EXACTKEYWORD,"Quality of Life") OR LIMIT-TO 
(EXACT KEYWORD, "Heart Assist Device") OR LIMIT-TO (EXACT KEYWORD, "Heart-Assist Devices")) AND (LIMITTO(LANGUAGE, "English") OR LIMIT-TO (LANGUAGE, "Portuguese") OR LIMIT-TO (LANGUAGE, "Spanish")).

O processo de seleção dos estudos foi dividido em duas etapas para evitar o surgimento de vieses. Primeiramente, os estudos iniciais foram inseridos em uma tabela no Programa Microsoft Excel® 2016 da Microsoft ${ }^{\circledR}$ para uma realização de uma leitura em pares criteriosa dos títulos e resumos para o estabelecimento dos artigos que atendessem aos critérios de inclusão supracitados, onde dois avaliadores, de forma independente, baseado nos títulos dos estudos, selecionaram possíveis artigos que podem cumprir os critérios de inclusão amostral.

Posteriormente, os mesmos leram os estudos na íntegra onde a amostra final dos artigos correspondeu pelos estudos selecionados após uma análise minuciosa e detalhada dos estudos inicialmente selecionados para uma posterior análise à luz da literatura. Houve a necessidade do envolvimento de um terceiro avaliador por haver divergência durante uma das etapas de seleção.

Os artigos foram organizados por semelhanças e, com a análise dos dados obtidos, as informações foram contidas em um banco de dados feito no Programa Excel® tendo seu embasamento fundamentado na literatura atualizada utilizando estudos, diretrizes e guidelines acerca da temática. Os estudos foram posteriormente categorizados em um banco de dados conforme os autores, ano de publicação, resultados e conclusões.

Além disso, classificou-se o nível de evidência (NE) dos estudos através dos seguintes critérios: 1) Revisões sistemáticas ou metanálise de ensaios clínicos randomizados relevantes; 2) Evidências provenientes de, pelo menos um, ensaio clínico randomizado controlado bem delimitado; 3) Ensaios clínicos bem delimitados sem randomização; 4) Estudos de coorte e de caso-controle; 5) Revisões sistemáticas de estudos descritivos e qualitativos; 6) Evidências de um único estudo descritivo ou qualitativo; 7) Opiniões de autoridades ou relatório de comitês de especialistas (Sampaio et al., 2020). A categorização dos estudos foi sumarizada em uma planilha para a apreciação. Compuseram a amostra 21 artigos.

\section{Resultados}

Para uma melhoria no relato de revisões sistemáticas e auxílio na seleção de estudos (Galvão, Pansani \& Harrad, 2015), aplicou-se o Preferred Reporting Items for Systematic Reviews and Meta-Analyses (PRISMA) nos 21 artigos incluídos nesta revisão, conforme fluxograma apresentado na Figura 1. 
Figura 1: Fluxograma do processo de seleção dos artigos utilizando o modelo PRISMA

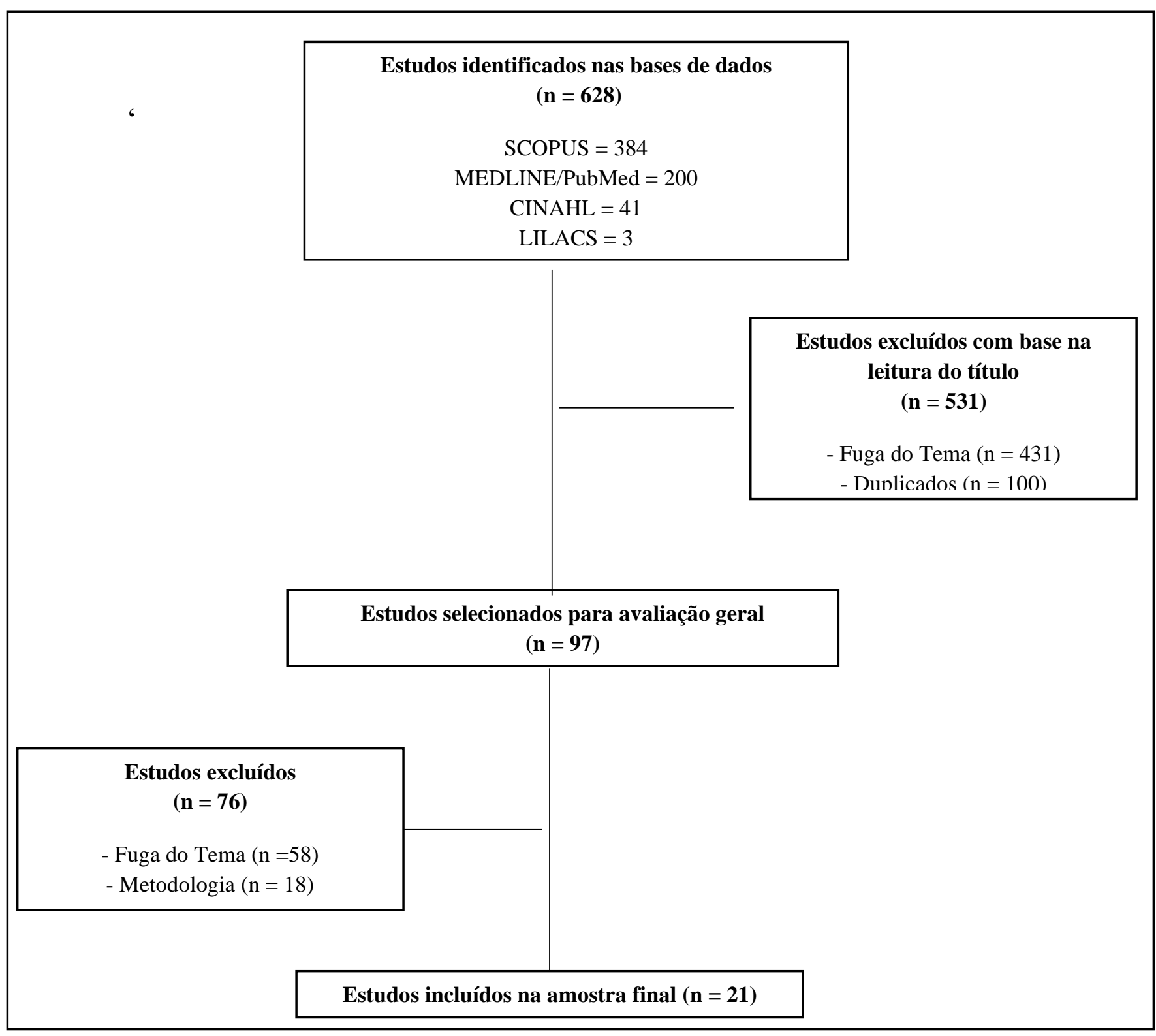

Fonte: Autores (2021).

A busca foi dividida em dois momentos. O primeiro consistiu na busca inicial nas bases de dados utilizando os descritores elencados para compor o quantitativo primário de artigos onde foram encontrados inicialmente 628 estudos nas bases de dados elencadas, sendo 384 na SCOPUS; 200 na National Library of Medicine and National Institutes of Health (MEDLINE)/PubMed; 41 CINAHL e 3 na LILACS.

Os títulos de todos os estudos foram agrupados em uma planilha no Excel contendo duas colunas para que os dois examinadores, separadamente, indicassem se o artigo deveria ou não ser lido na íntegra, sendo que 70 estudos tiveram avaliação divergente entre os dois examinadores, necessitando de uma avaliação de um terceiro avaliador com expertise. Após a utilização dos critérios de exclusão estabelecidos, 97 estudos foram determinados para uma análise completa.

Os 21 estudos pertencentes à amostra final não tiveram definição de recorte temporal e corresponderam à última década, compreendendo trabalhos dos anos de 2010 a 2020, sendo 2018 o ano mais expressivo com 28,5\% (n = 6) dos trabalhos publicados. Os anos de 2010, 2014 e 2019 contaram com dois artigos cada; 2016 e 2017 com um estudo; 2015 com 
uma expressividade de quatro manuscritos e 2020 com dois trabalhos para finalizar a amostra. Estudos publicados nos anos de 2011, 2012 e 2013 não foram contemplados no rol de artigos.

Nessa busca, todos as publicações foram disponibilizadas na língua inglesa reiterando assim, o fato de a amostra ter sido publicizada completamente em periódicos internacionais. Quanto ao nível de evidência, reitera-se o predomínio, como percurso metodológico na amostra, o estudo transversal. Estudos de coorte e ensaio clínico randomizado, que possuem um maior nível de evidência, também fizeram parte da amostra. Para completar, análises de estudos observacionais e estudos longitudinais e descritivos fizeram-se pertencentes na seleção final das publicações.

Para uma melhor organização, após a leitura criteriosa dos estudos da amostra, eles foram listados e organizados em um banco de dados no programa Microsoft Excel® contendo a codificação dos artigos (nomeados de E1 ao E21), autores, ano, nível de evidência, objetivo do estudo, resultados e conclusão. Os dados estão contidos na Tabela 1 a seguir.

Tabela 1: Amostra da revisão integrativa com base na codificação, título, autores, ano e nível de evidência.

\begin{tabular}{|c|c|c|c|c|}
\hline Código & Título & Autores & Ano & $\begin{array}{c}\text { Nível de } \\
\text { Evidência }\end{array}$ \\
\hline 1 & $\begin{array}{c}\text { Coping, Mood, Quality of Life, and Outcomes in Recipients of Left Ventricular } \\
\text { Assist Devices: A Cluster Analysis }\end{array}$ & Modica M. et al & 2019 & 4 \\
\hline 2 & $\begin{array}{c}\text { Quality of Life and Treatment Preference for VAD therapy in Ambulatory } \\
\text { Advanced Heart Failure: A Report from the REVIVAL Study }\end{array}$ & Stehlik J. et al & 2020 & 6 \\
\hline 3 & $\begin{array}{l}\text { Quality of Life and Influential Factors in Patients } \\
\text { Implanted With a Left Ventricular Assist Device }\end{array}$ & Kato N. et al. & 2015 & 6 \\
\hline 4 & $\begin{array}{c}\text { A longitudinal comparison of health-related quality of life in rural and urban } \\
\text { recipients of left ventricular assist devices }\end{array}$ & Alonso W.W. et al. & 2020 & 6 \\
\hline 5 & $\begin{array}{c}\text { Quality of Life and Emotional Distress Early After Left Ventricular Assist Device } \\
\text { Implant: A Mixed-Method Study }\end{array}$ & Modica M. et al & 2015 & 6 \\
\hline 6 & $\begin{array}{c}\text { Preparedness and Mutuality Affect Quality of Life for Patients With Mechanical } \\
\text { Circulatory Support and Their Caregivers: A Qualitative Analysis }\end{array}$ & Magasi S. et al & 2019 & 6 \\
\hline 7 & $\begin{array}{c}\text { The Relationship of Anxiety, Depression, and Quality of Life in Adults With Left } \\
\text { Ventricular Assist Devices }\end{array}$ & Casida J.M. et al. & 2018 & 6 \\
\hline 8 & $\begin{array}{c}\text { Quality of Life, Depression, and Anxiety in Ventricular Assist Device Therapy: } \\
\text { Longtidunal Outcomes for Patients and Family Caregivers }\end{array}$ & Bidwell J.T. et al & 2018 & 6 \\
\hline 9 & $\begin{array}{c}\text { Patient and Caregiver Determinants of Patient Quality of Life and Caregiver } \\
\text { Strain in Left Ventricular Assist Device Therapy }\end{array}$ & Bidwell J.T. et al & 2018 & 6 \\
\hline 10 & $\begin{array}{c}\text { Quality of life of advanced chronic heart failure: medical care, mechanical } \\
\text { circulatory support and transplantation }\end{array}$ & $\begin{array}{l}\text { Emina A. Rogers C. } \\
\text { A. Bannerc N. R. }\end{array}$ & 2016 & 4 \\
\hline 11 & $\begin{array}{c}\text { Quality of life and functional capacity outcomes in the MOMENTUM } 3 \text { trial at } 6 \\
\text { months: A call for new metrics for left ventricular assist device patients }\end{array}$ & Cowger J. A. & 2018 & 2 \\
\hline 12 & $\begin{array}{c}\text { What Really Matters? Understanding Quality of Life Determinants Impacting } \\
\text { Ventricular Assist Device Stakeholders }\end{array}$ & Ko K. H. K. et al & 2020 & 6 \\
\hline 13 & $\begin{array}{l}\text { Sexual activity in patients with left ventricular assist devices and their partners: } \\
\text { impact of the device on quality of life, anxiety and depression }\end{array}$ & Kugler C. et al & 2018 & 6 \\
\hline 14 & $\begin{array}{c}\text { Social Support Moderates the Relationship between Perceived Stress and Quality } \\
\text { of Life in Patients with a Left Ventricular Assist Device }\end{array}$ & Abshire M. et al & 2018 & 6 \\
\hline 15 & $\begin{array}{l}\text { Cognition and adherence are self-management factors predicting the quality of } \\
\text { life of adults living with a left ventricular assist device }\end{array}$ & Casida J. M. et al & 2017 & 6 \\
\hline 16 & $\begin{array}{c}\text { Health-related quality of life in mechanical circulatory support: Development of a } \\
\text { new conceptual model and items for self-administration }\end{array}$ & Grady K. L. et al. & 2015 & 6 \\
\hline 17 & Sexual Concerns of Patients With Implantable Left Ventricular Assist Devices & Merle P. et al & 2015 & 6 \\
\hline 18 & $\begin{array}{c}\text { Effect of Left Ventricular Assist Device Implantation and Heart Transplantation } \\
\text { on Habitual Physical Activity and Quality of Life }\end{array}$ & $\begin{array}{l}\text { Jakovljevic D. G. et } \\
\text { al. }\end{array}$ & 2014 & 6 \\
\hline 19 & $\begin{array}{l}\text { Overall Quality of Life Improves to Similar Levels after Mechanical Circulatory } \\
\text { Support Regardless of Severity of Heart Failure before Implantation }\end{array}$ & Grady K. et al & 2014 & 6 \\
\hline 20 & $\begin{array}{c}\text { Continuous Flow Left Ventricular Assist Device Improves Functional Capacity } \\
\text { and Quality of Life of Advanced Heart Failure Patients }\end{array}$ & Rogers J. G. et al & 2010 & 6 \\
\hline 21 & $\begin{array}{c}\text { Quality of Life and Functional Status in patients surviving } 12 \text { months after Left } \\
\text { Ventricular Assist Device Implantation }\end{array}$ & Allen J. G. et al & 2010 & 4 \\
\hline
\end{tabular}

Fonte: Autores (2021).

\section{Discussão}

O uso de DAV na terapêutica visa melhorar a qualidade de vida dos pacientes, que deve ser entendida de forma multifatorial avaliando todas as condições envolvidas. Mesmo com limitações funcionais próprias da condição clínica, estudos 
apontam que os DACM trazem melhor qualidade de vida comparado ao tratamento conservador para a IC avançada (Fernandes et al., 2020).

A utilização de um DAV traz alterações significativas na qualidade de vida dos pacientes nos âmbitos sociais, físico, psicológico e no bem-estar geral. Segundo a Diretriz Brasileira de Assistência Circulatória Mecânica (2018), para a indicação do dispositivo é realizada uma complexa avaliação do paciente buscando aferir fatores como: histórico, compreensão da condição clínica, função cognitiva, terapêutica, motivação, medo, enfrentamento, adesão ao tratamento farmacológico e não farmacológico, suporte social e emocional, dentre outros.

Estudos destacam que pacientes com IC avançada refratária ao tratamento em uso de DAV apresentam uma qualidade de vida superior comparada aos indivíduos com IC apenas com a terapêutica clínica otimizada (Bidwell et al., 2017; Emin, Rogers \& Banner, 2016). Alonso e colaboradores (2020) trazem que antes da instalação do dispositivo, os pacientes apresentam constantes descompensações clínicas decorrentes da sintomatologia característica da insuficiência cardíaca e, após o recebimento da terapia, existe uma melhora nos sintomas que acarreta em melhora da qualidade de vida.

Estudo prospectivo e observacional realizado por Kato et al. (2017) com 33 japoneses após três meses do implante de DAV trouxe como principal achado a melhoria da QV após o implante. Somado a esse fator, Bidwell e autores (2017) também apontaram melhorias grandes e estatisticamente significantes na qualidade da vida de pacientes com dispositivo ao comparar o período pré ao pós-DAV.

Em contrapartida, as mudanças do estilo de vida que são imprescindíveis para viver com um dispositivo em segurança pode acabar trazendo sentimentos negativos ao paciente, principalmente relacionados ao processo de adaptação, risco de aumento de hospitalizações e potenciais complicações como Acidente Vascular Cerebral (AVC), sangramentos, arritmias e infecções (Cowger et al., 2018; Ko et al., 2020).

Uma pesquisa realizada por Grady et al. (2014) com adultos com IC avançada que receberam um suporte circulatório mecânico, aponta que em um ano após a cirurgia os níveis de QV tendem a melhorar, porém cerca de 30\% dos pacientes relatam, nesse período, episódios de dor, desconforto, ansiedade e depressão que impactam na qualidade de vida. Salienta-se que uma qualidade de vida considerada ruim é fator preditor de um prognóstico ruim onde pode acarretar em óbito, readmissão hospitalar, descompensação hemodinâmica e, inclusive, transplante cardíaco de urgência.

A convivência com um DAV tem uma correlação direta com a cognição e o estado psicológico do paciente porque o artefato traz um impacto na qualidade de vida, visto que o seu manejo adequado é complexo e pode implicar na não obtenção de um nível satisfatório de autonomia. Além disso, fatores como a dependência do cuidado de terceiros e/ou de serviços de saúde especializados corroboram para uma limitação na vida social (Modica et al., 2015).

Estudo observacional proposto por Casida e colaboradores (2017) que avaliou o autocuidado de 87 pacientes com $\mathrm{DAV}$, reiterou que um nível alto de eficácia do dispositivo está diretamente associado à adesão terapêutica das recomendações de cuidado, ou seja, quando um indivíduo possui uma capacidade maior para aprender, reter informações e mostrar domínio, acarreta em uma menor dependência direta de cuidado ao artefato e melhoria na qualidade de vida.

Apesar dos DAV melhorarem significativamente a sobrevida dos pacientes, existem dificuldades que limitam a adesão da terapia como o manejo da tecnologia, mudança nos hábitos de vida, consultas de saúde periódicas, cuidados específicos, troca de baterias, dentre outros (Kilic, 2015).

Tendo em vista a complexidade do tratamento, um ponto fundamental para o sucesso da terapêutica é o suporte social e/ou familiar e a rede de apoio que melhoram muito o status emocional de angústias, medo e preocupações (Bidwell et al., 2018). O implante de um DAV representa uma grande mudança que gera repercussões psicológicas. Compreender os sentimentos dos indivíduos e avaliar sua rede de apoio é de extrema importância para a qualidade de vida do paciente (Asleh et al., 2019). 
A literatura nos traz a dualidade acerca da qualidade de vida de pacientes em uso de DAV. Existem fatores positivos e negativos relatados nos estudos, entretanto, deve-se considerar todo o contexto envolvido.

\section{Considerações Finais}

Entende-se que um cuidado fundamental é melhorar a qualidade de vida do paciente o que gera um grande impacto no seu cotidiano. É imprescindível que a equipe multiprofissional aproprie-se acerca de novas tecnologias que envolvem o cuidado ao paciente cardiopata para fornecer uma assistência direcionada e efetiva na promoção da qualidade de vida.

Os estudos apontam que os pacientes em uso de DAV apresentam melhora da sintomatologia, diminuem a descompensação clínica decorrente da insuficiência cardíaca e, consequentemente, melhoram a qualidade de vida. Compreender esse aspecto repercute positivamente não apenas na clínica do paciente, mas também na adesão ao tratamento proposto, no autocuidado e, inclusive, no protagonismo da utilização correta do dispositivo utilizado.

Apesar da escassez de estudos sobre a temática e obstáculos que impossibilitam essa alternativa terapêutica, os dispositivos já se encontram no Brasil e constituem-se de estratégias eficazes frente a essa condição. Fomentar o conhecimento acerca da utilização de DAV envolve ampliar os horizontes para aqueles que se encontram na fila de espera por um transplante cardíaco.

\section{Referências}

Abshire, M., Prichard, R., Cajita, M., DiGiacomo, M., \& Dennison Himmelfarb, C. (2016). Adaptation and coping in patients living with an LVAD: A metasynthesis. Heart \& lung: the journal of critical care, 45(5), 397-405. https://doi.org/10.1016/j.hrtlng.2016.05.035

Alonso, W. W., Faulkner, K. M., Pozehl, B. J., Hupcey, J. E., Kitko, L. A., \& Lee, C. S. (2020). A longitudinal comparison of health-related quality of life in rural and urban recipients of left ventricular assist devices. Research in nursing \& health, 43(4), 396-406. https://doi.org/10.1002/nur.22052

Asleh, R., Schettle, S. S., Khan, F. W., \& Kushwaha, S. S. (2019). Left ventricular assist devices as destination therapy in stage D heart failure. Journal of geriatric cardiology: JGC, 16(8), 592-600. https://doi.org/10.11909/j.issn.1671-5411.2019.08.009

Ayub-Ferreira, S. M., Souza, J. D., Neto, Almeida, D. R., Biselli, B., Avila, M. S., Colafranceschi, A. S., Stefanello, B., Carvalho, B. M., Polanczyk, C. A., Galantini, D. R., Bocchi, E. A., Chamlian, E. G., Hojaij, E. M., Gaiotto, F. A., Pinton, F. A., Jatene, F. B., Ramires, F., Atik, F. A., Figueira, F., Bacal, F., \& Issa, V. S. (2016). Diretriz de Assistência Circulatória Mecânica da Sociedade Brasileira de Cardiologia. Arquivos brasileiros de cardiologia, 107(2 Suppl 2), 1-33. https://doi.org/10.5935/abc.20160128

Barbosa, R. R., Franklin, R. V., Stefenoni, A. V., Moraes, V. D., Jacques, T. M., Serpa, R. G., Calil, O. A., \&Barbosa, L. F. M. (2014). Análise da qualidade de vida em homens e mulheres portadores de insuficiência cardíaca. Revista Brasileira de Cardiologia, $27(2)$, 97-103. https://www.onlineijcs.com/detalhes/15/quality-of-life-analysis-among-men-and-women-with-heart-failure

Bidwell, J. T., Lyons, K. S., Mudd, J. O., Gelow, J. M., Chien, C. V., Hiatt, S. O., Grady, K. L., \& Lee, C. S. (2017). Quality of Life, Depression, and Anxiety in Ventricular Assist Device Therapy: Longitudinal Outcomes for Patients and Family Caregivers. The Journal of cardiovascular nursing, 32(5), 455-463. https://doi.org/10.1097/JCN.0000000000000378

Bidwell, J. T., Lyons, K. S., Mudd, J. O., Grady, K. L., Gelow, J. M., Hiatt, S. O., Chien, C. V., \& Lee, C. S. (2018). Patient and Caregiver Determinants of Patient Quality of Life and Caregiver Strain in Left Ventricular Assist Device Therapy. Journal of the American Heart Association, 7(6), e008080. https://doi.org/10.1161/JAHA.117.008080

Casida, J. M., Wu, H. S., Abshire, M., Ghosh, B., \& Yang, J. J. (2017). Cognition and adherence are self-management factors predicting the quality of life of adults living with a left ventricular assist device. The Journal of heart and lung transplantation, 36(3), 325-330. https://doi.org/10.1016/j.healun.2016.08.023

Cerqueira, A. C. D. R., Cardoso, M. V. L. M. L., Viana, T. R. F., \&Lopes, M. M. C. O. (2018). Revisão integrativa da literatura: sono em lactentes que frequentam creche. Revista Brasileira de Enfermagem, 71(2), 424-430. https://doi.org/10.1590/0034-7167-2016-0480.

Cowger, J. A., Naka, Y., Aaronson, K. D., Horstmanshof, D., Gulati, S., Rinde-Hoffman, D., Pinney, S., Adatya, S., Farrar, D. J., Jorde, U. P., \& MOMENTUM 3 Investigators (2018). Quality of life and functional capacity outcomes in the MOMENTUM 3 trial at 6 months: A call for new metrics for left ventricular assist device patients. The Journal of heart and lung transplantation, 37(1), 15-24. https://doi.org/10.1016/j.healun.2017.10.019

Emin, A., Rogers, C. A., Banner, N. R., \& Steering Group, UK Cardiothoracic Transplant Audit (2016). Quality of life of advanced chronic heart failure: medical care, mechanical circulatory support and transplantation. European journal of cardio-thoracic surgery, 50(2), 269-273. https://doi.org/10.1093/ejcts/ezw054 
Fernandes, A. D. F., Fernandes, G. C., Mazza, M. R., Knijnik, L. M., Fernandes, G. S., Vilela, A. T., Badiye, A., \& Chaparro, S. V. (2020). Insuficiência cardíaca no Brasil subdesenvolvido: análise de tendência de dez anos. Arquivos Brasileiros de Cardiologia, 114(2), 222-231 https://doi.org/10.36660/abc.20180321

Galvão, T. F., Pansani, T. S. A., \& Harrad, D. (2015). Principais itens para relatar revisões sistemáticas e meta-análises: a recomendação PRISMA. Epidemiologia e Serviços de Saúde, 24(2), 335-342 https://doi.org/10.5123/S1679-49742015000200017

Garlet, A. B., Cardoso, D. M., Santos, T. D., Pereira S. N. \& Albuquerque, I. M. (2017). Relação entre classe funcional e fração de ejeção do ventrículo esquerdo em pacientes com doença coronariana candidatos à reabilitação cardíaca. Scientia Medica, 27(3) https://doi.org/10.15448/1980-6108.2017.3.26652

Grady, K. L., Naftel, D., Stevenson, L., Dew, M. A., Weidner, G., Pagani, F. D., Kirklin, J. K., Myers, S., Baldwin, T., \& Young, J. (2014). Overall quality of life improves to similar levels after mechanical circulatory support regardless of severity of heart failure before implantation. The Journal of heart and lung transplantation, 33(4), 412-421. https://doi.org/10.1016/j.healun.2013.10.017

Jakovljevic, D. G., Yacoub, M. H., Schueler, S., MacGowan, G. A., Velicki, L., Seferovic, P. M., Hothi, S., Tzeng, B. H., Brodie, D. A., Birks, E., \& Tan, L. B. (2017). Left Ventricular Assist Device as a Bridge to Recovery for Patients With Advanced Heart Failure. Journal of the American College of Cardiology, 69(15), 1924-1933. https://doi.org/10.1016/j.jacc.2017.02.018

Kato, N. P., Okada, I., Imamura, T., Kagami, Y., Endo, M., Nitta, D., Fujino, T., Muraoka, H., Minatsuki, S., Maki, H., Inaba, T., Kinoshita, O., Nawata, K., Hatano, M., Yao, A., Kyo, S., Ono, M., Jaarsma, T., \& Kinugawa, K. (2015). Quality of Life and Influential Factors in Patients Implanted With a Left Ventricular Assist Device. Circulation journal, 79(10), 2186-2192. https://doi.org/10.1253/circj.CJ-15-0502

Kilic A. (2015). The future of left ventricular assist devices. Journal of thoracic disease, 7(12), 2188-2193. https://doi.org/10.3978/j.issn.20721439.2015.11.01

Ko, K., Straker, K., Dunn, J. L., \& Wrigley, C. (2020). What Really Matters? Understanding Quality of Life Determinants Impacting Ventricular Assist Device Stakeholders. ASAIO journal (American Society for Artificial Internal Organs), 66(6), 626-636. https://doi.org/10.1097/MAT.0000000000001175

Maciver, J., \& Ross, H. J. (2012). Quality of life and left ventricular assist device support. Circulation, 126(7), 866-874. https://doi.org/10.1161/CIRCULATIONAHA.111.040279

Modica, M., Ferratini, M., Torri, A., Oliva, F., Martinelli, L., De Maria, R., \& Frigerio, M. (2015). Quality of life and emotional distress early after left ventricular assist device implant: a mixed-method study. Artificial organs, 39(3), 220-227. https://doi.org/10.1111/aor.12362

Rohde, L., Montera, M. W., Bocchi, E. A., Clausell, N. O., Albuquerque, D. C., Rassi, S., Colafranceschi, A. S., Freitas, A. F., Junior, Ferraz, A. S., Biolo, A., Barretto, A., Ribeiro, A., Polanczyk, C. A., Gualandro, D. M., Almeida, D. R., Silva, E., Figueiredo, E. L., Mesquita, E. T., Marcondes-Braga, F. G., \& Martins, W. A. (2018). Diretriz Brasileira de Insuficiência Cardíaca Crônica e Aguda. Arquivos brasileiros de cardiologia, 111(3), 436-539. https://doi.org/10.5935/abc.20180190

Sampaio, S. M. L., Sousa, A. A. S., Queiroz, I. B. S., Luna, C. A., Gurgel, L. C., Luna, T. B., Sousa, C. M. S., Sousa, F. R. S., Luz, D. C. R. P., \& Santana, W. J. (2020). Atuação de enfermagem frente ao atendimento ao paciente hipertenso. Revista Eletrônica Acervo Saúde, 50. https://doi.org/10.25248/reas.e3526.2020

Santos, M. C., Pimenta, C. A. M., \& Nobre, M. R. C. (2007). A estratégia PICO para a construção da pergunta de pesquisa e busca de evidências. Revista Latino-Americana de Enfermagem, 15(3) https://doi.org/10.1590/S0104-11692007000300023

Shah, S. R., Issa Najim, N., Shah, S. A., Shahnawaz, W., \& Ahmed Jangda, M. (2018). Using left ventricular assist devices in advanced heart failure patients. Journal of community hospital internal medicine perspectives, 8(6), 357-359. https://doi.org/10.1080/20009666.2018.1536240

Slaughter, M. S., \& Singh, R. (2012). The role of ventricular assist devices in advanced heart failure. Revista espanola de cardiologia (English ed.), 65(11), 982-985. https://doi.org/10.1016/j.recesp.2012.02.030

Sousa, M. M., Oliveira, J. S., Soares, M. J. G. O., Bezerra, S. M. M. S., Araújo, A. A., \& Oliveira, S. H. S. (2017). Associação das condições sociais e clínicas à qualidade de vida de pacientes com insuficiência cardíaca. Revista Gaúcha de Enfermagem, 38(2):e65885. https://doi.org/10.1590/19831447.2017.02.65885

Souza, M. T., Silva, M. D., \& Carvalho, R. (2010). Revisão integrativa: o que é e como fazer. Einstein, 8(1): 102-106. https://doi.org/10.1590/S167945082010RW1134 